\title{
Effects of Forest Therapy on Depressive Symptoms among Adults: A Systematic Review
}

\author{
Insook Lee ${ }^{1}$, Heeseung Choi ${ }^{1, *}$, Kyung-Sook Bang ${ }^{1}$, Sungjae Kim ${ }^{1}$, MinKyung Song ${ }^{2}$ \\ and Buhyun Lee ${ }^{2}$ \\ 1 Faculty of College of Nursing, The Research Institute of Nursing Science, Seoul National University, \\ Seoul 03080, Korea; lisook@snu.ac.kr (I.L.); ksbang@snu.ac.kr (K.-S.B.); sungjae@snu.ac.kr (S.K.) \\ 2 College of Nursing, Seoul National University, Seoul 03080, Korea; mk0408@snu.ac.kr (M.S.); \\ bhyunlee@snu.ac.kr (B.L.) \\ * Correspondence: hchoi20@snu.ac.kr; Tel.: +82-2-740-8850
}

Academic Editor: Paul B. Tchounwou

Received: 24 December 2016; Accepted: 9 February 2017; Published: 20 March 2017

\begin{abstract}
This study systematically reviewed forest therapy programs designed to decrease the level of depression among adults and assessed the methodological rigor and scientific evidence quality of existing research studies to guide future studies. This systematic review was conducted in accordance with the Preferred Reporting Items for Systematic Reviews and Meta-Analyses guidelines. The authors independently screened full-text articles from various databases using the following criteria: (1) intervention studies assessing the effects of forest therapy on depressive symptoms in adults aged 18 years and older; (2) studies including at least one control group or condition; (3) peer-reviewed studies; and (4) been published either in English or Korean before July 2016. The Scottish Intercollegiate Guideline Network measurement tool was used to assess the risk of bias in each trial. In the final sample, 28 articles (English: 13, Korean: 15) were included in the systematic review. We concluded that forest therapy is an emerging and effective intervention for decreasing adults' depression levels. However, the included studies lacked methodological rigor. Future studies assessing the long-term effect of forest therapy on depression using rigorous study designs are needed.
\end{abstract}

Keywords: systematic review; forest therapy; depression; adults

\section{Introduction}

Forest therapy or "forest bathing" refers to visiting a forest or engaging in various therapeutic activities in a forest environment to improve one's health and wellbeing [1,2]. Societies have been urbanizing rapidly and more people reside in an urban environment with limited access to nature; therefore, diverse efforts including political and landscaping efforts have been made to make nature more accessible [3]. With an increasing awareness of health benefits of forest therapy, it has been implemented on diverse population [1]. Particularly, the psychological benefits of forest therapy have received special attention as people residing in urban environments have been reported to be at an increased risk of prolonged exposure to stressful situations and mental health problems [4-6]. Compared to control groups, forest therapy significantly improves adults' mental health by decreasing stress, depression, anxiety, and anger levels [7].

A systematic review summarizes the results of the available research studies and provides synthesized evidence on the effectiveness of those studies [8]. It enables researchers to identify the current state of the science, areas for future researchers to improve upon, and provides strong evidence for up-to-date practices and policy developments [9]. It is also beneficial for emerging topics that require systematic evaluation and synthesis of the evidence quality (e.g., feasibility and effectiveness 
of intervention) as well as well-established areas of research with accumulated scientific evidence that need be updated regularly.

Despite the increased attention to the various health benefits of forest therapy, until now, systematic reviews of the body of evidence for the effectiveness of forest therapy on mental health have not been conducted. A clearer and comprehensive understanding of the effectiveness of forest therapy on mental health is important for further refinement of forest therapy programs. Among the several mental health outcomes included in the forest therapy research, our paper will focus on depression. Depression is the leading cause of disability; approximately 350 million ( $5 \%$ of the world's population) suffer from this debilitating disorder [10]. The specific aims of this study were to: (1) provide a broad overview and synthesize the evidence on the usefulness of forest therapy to improve the level of depressive symptoms in adults; and (2) assess the methodological rigor and scientific evidence quality of existing research studies to guide future studies evaluating the effects of forest therapy on adults' experiencing depressive symptoms. In the present review, forest therapy was defined as visiting a forest or engaging in various therapeutic activities in a forest environment to improve one's health and wellbeing [1,2].

\section{Methods}

\subsection{Literature Search}

This systematic review was conducted in accordance with the Preferred Reporting Items for Systematic Reviews and Meta-Analyses (PRISMA) guidelines. We searched PubMed, EMBASE, Cumulative Index to Nursing and Allied Health, PsycARTICLES, Korean Studies Information Service System, Research Information Sharing Service, and DBpia to identify relevant studies published until July 2016. The search terms were chosen from the USNLM Institutes of Health list of Medical Subject Headings for 2015. Search terms included "trees", "forests", "wood", "affect", "depression", "emotions" and "depressive disorder". Search terms used to identify relevant studies for the review are listed in Appendix A, Table A1.

\subsection{Selection Criteria}

The initial eligibility assessment was conducted by one author by reviewing the title and abstracts. Then, two authors (MinKyung Song and Buhyun Lee) independently screened the full text versions of 66 articles using the following criteria: (1) intervention studies assessing the effects of forest therapy on depression among adults aged 18 years and older; (2) studies including at least one control group or condition; (3) peer-reviewed studies; and (4) been published either in English or Korean.

\subsection{Data Extraction}

The four authors (Heeseung Choi, Kyung-Sook Bang, MinKyung Song, and Buhyun Lee) independently performed the data extraction. The following data were extracted from each study: first author, date and place of publication, study design, sample size, setting, ethical consideration, participants' characteristics, number of participants enrolled, summary of the intervention and control conditions, measures, reported outcomes, and risk of bias. The extracted data were input into standardized MS word (Microsoft Corporation, Seattle, WA, USA) files. Any disagreements were resolved by discussion between the authors.

\subsection{Quality Assessment Tool}

The Scottish Intercollegiate Guideline Network (SIGN) measurement tool (Healthcare Improvement Scotland, Edinburgh, Scotland) was used to assess the risk of bias in each study included in this review. The SIGN was developed in 1993 to improve the quality of health care for patients in Scotland by reducing the variation in practice and outcome, through the development and dissemination of national clinical guidelines containing recommendations for effective practice based on current evidence [11]. 
Using the SIGN, we evaluated the internal validity and risk of bias of the study and assigned values of "high quality (++)," "acceptable (+)," "low quality (-)," or "unacceptable-reject (0)" to each study. The risk of bias was evaluated independently by four reviewers (Heeseung Choi, Kyung-Sook Bang, MinKyung Song, and Buhyun Lee) and any disagreements were resolved through a consensus process.

\section{Results}

\subsection{Study Identification and Selection}

Records ( $\mathrm{N}=8355)$, including 4399 records published in English and 3956 records published in Korean, were retrieved from the initial database searches. These search results were imported using EndNote X7 and 1516 duplicates (1356 English articles and 160 Korean articles) were removed. A detailed flow diagram of the screening process is shown in Figure 1. After excluding an additional 6773 records based on the review of the study titles and abstracts, the remaining 21 articles published in English and 45 articles published in Korean were assessed for eligibility. Many articles were excluded because those studies addressed topics that were not relevant to forest therapy, such as tree analysis (i.e., classification and regression tree analysis), biliary/bronchial tree, forest modeling, and forest fragmentation. Finally, 28 articles (English: 13, Korean: 15) were included in the present systematic review.

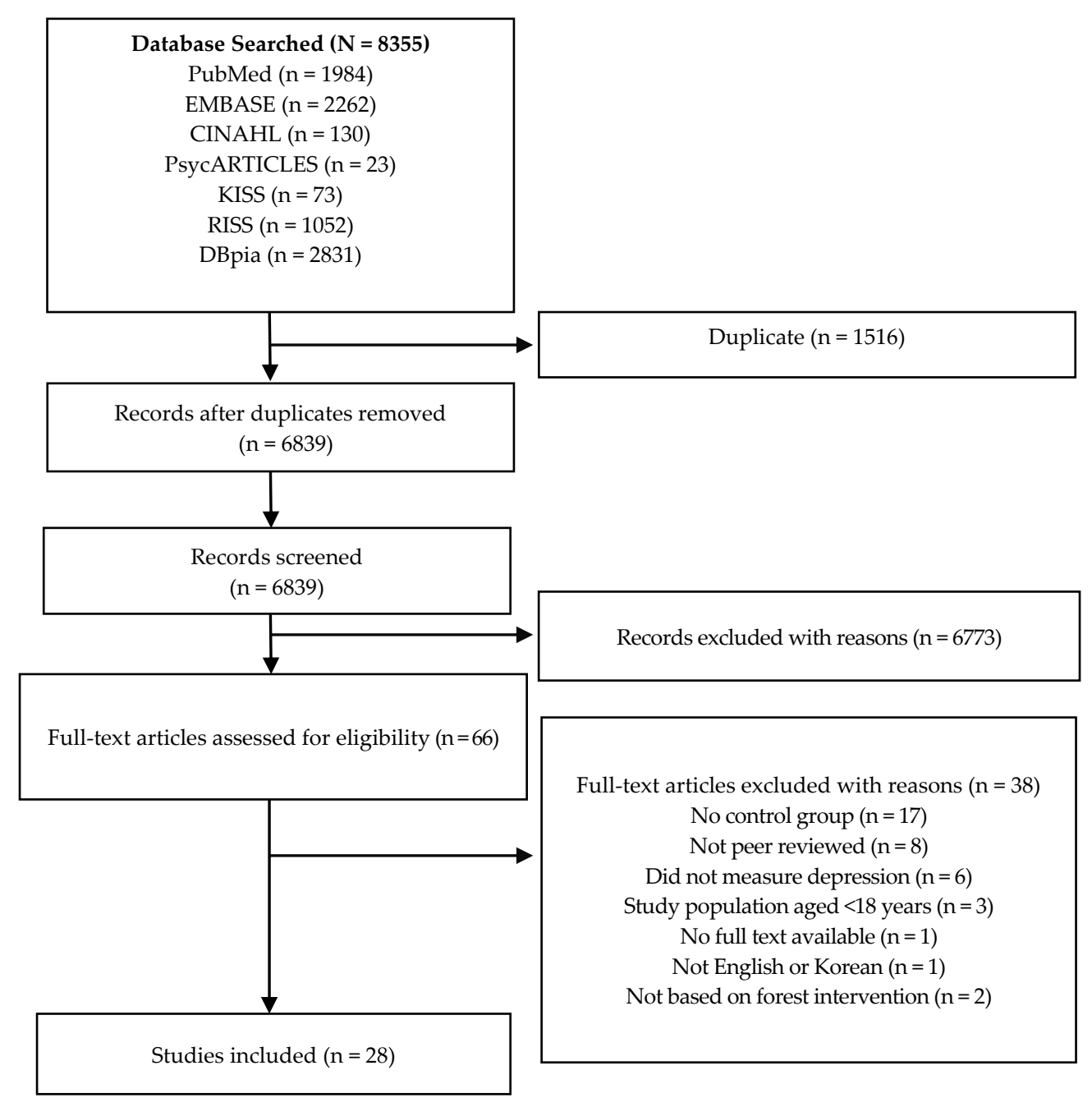

Figure 1. Preferred Reporting Items for Systematic Reviews and Meta-Analyses (PRISMA) Flow Diagram of the Screening Process. 


\subsection{Study Characteristics}

The general characteristics of the studies included in this review are summarized in Table 1. And summary of the studies included in this review are presented in Tables 2 and 3. The selected studies were published between 1996 and 2016, and 24 of the 28 studies were published within the last five years. All the studies were conducted in Asian countries (Korea, Japan, and China) except one, which was conducted in the United Kingdom. Sixteen studies were conducted on healthy adults and the rest of the studies $(n=12)$ were conducted on adults with various health problems such as hypertension, cancer, and mental disorders. Among the 12 studies conducted for adults with health problems, six studies targeted psychiatric patients [12-17]; however, only one study [17] was conducted with patients with major depressive disorder. Two studies $[12,15]$ were conducted for psychiatric patients with various diagnoses such as substance use disroders, schizophrenia, other psychotic disorders, mood disorders, and anxiety disorders. These two studies, however, did not specify what percentage of their samples were patients with major depressive disorder. Other studies targeted specific diagnoses such as Hwa-Byung [13], neurocognitive disorders [14], and alcoholism [16].

Regarding the study design, 11 studies used a crossover trial design and only six of the studies [16,18-22] used a randomized controlled trial (RCT) design. Four out of six RCT design studies were conducted with adults with health problems. The most common types of control condition used in the non-equivalent control group design studies were "normal daily routines" for healthy adults (five out of six studies) and "treatment-as-usual" for adults with health problems (three out of five studies).

The sample size ranged from 11 [23] to 92 [16]; for almost $43 \%$ of the studies, the sample size was less than 20. Furthermore, about one third of the studies (28.5\%) did not follow the ethical protocol such as being reviewed and approved by the Institutional Review Board (IRB).

Table 1. General Characteristics of Included Studies $(\mathrm{N}=28)$.

\begin{tabular}{ccc}
\hline Variables & Categories & N (\%) \\
\hline \multirow{2}{*}{ Participants } & Healthy adults & $16(57.1)$ \\
& Adults with health problems & $12(42.9)$ \\
\hline \multirow{2}{*}{ Publication year } & $\leq 2000$ & $1(3.6)$ \\
& $2001-2011$ & $3(10.7)$ \\
& $2012-2016$ & $24(85.7)$ \\
\hline \multirow{2}{*}{ Country } & Republic of Korea & $17(60.7)$ \\
& Japan & $7(25.0)$ \\
Study Design & China & $3(10.7)$ \\
& United Kingdom & $1(3.6)$ \\
\hline \multirow{2}{*}{ Sample size } & Non-equivalent control group design & $6(21.4)$ \\
& Crossover trial design & $11(39.3)$ \\
& $\leq 20$ & $11(39.3)$ \\
\hline \multirow{2}{*}{ Setting } & $21-50$ & $6(21.4)$ \\
& $51-100$ & $10(35.7)$ \\
& $>100$ & $0(0)$ \\
\hline \multirow{2}{*}{ Ethical consideration } & Community & $8(28.6)$ \\
& Hospital & $5(17.9)$ \\
& University & $9(32.1)$ \\
& Unknown & $6(21.4)$ \\
\hline \multirow{2}{*}{ Yed control trial design } & $20(71.4)$ \\
& No & $8(28.6)$ \\
\hline
\end{tabular}


Table 2. Summary of Included Studies for Healthy Adults $(\mathbf{N}=16)$.

\begin{tabular}{|c|c|c|c|c|c|c|c|c|c|}
\hline \multirow{3}{*}{ Authors (Year) } & \multirow{3}{*}{ Country } & \multirow{3}{*}{ Research Design } & \multicolumn{2}{|c|}{ Participants (N) } & \multirow{3}{*}{ Intervention(s) } & \multirow{3}{*}{ Control } & \multirow{2}{*}{\multicolumn{2}{|c|}{ Measurements }} & \multirow{3}{*}{ Outcomes } \\
\hline & & & \multirow{2}{*}{ Exp. } & \multirow{2}{*}{ Cont. } & & & & & \\
\hline & & & & & & & Self-Report Measures & Physiological Measures & \\
\hline \multirow[b]{2}{*}{ Bang (2016) [18] } & \multirow[b]{2}{*}{ Republic of Korea } & \multirow[b]{2}{*}{ RCT } & \multicolumn{2}{|c|}{ Office workers $(\mathrm{n}=45)$} & \multirow[b]{2}{*}{$\begin{array}{l}\text { Urban forest-walking program- } \\
\text { Twice a week for five weeks- } \\
\text { Urban forest walking }(40 \mathrm{~min}) \\
\text { and Rest }(10 \mathrm{~min})\end{array}$} & \multirow[b]{2}{*}{$\begin{array}{l}\text { Normal } \\
\text { daily routines }\end{array}$} & \multirow{2}{*}{$\begin{array}{l}\text { Health-Promoting } \\
\text { Lifestyle Profile } \\
\text { (HPLP) II } \\
\text { Beck Depression } \\
\text { Inventory (BDI) } \\
\text { General Health } \\
\text { Questionnaire/Quality } \\
\text { of life (GHQ/QL-12) }\end{array}$} & \multirow[b]{2}{*}{ Blood pressure (BP) } & $\begin{array}{l}\text { Physical activity level * } \\
\text { Health-Promoting behavior * }\end{array}$ \\
\hline & & & $\mathrm{n}=18$ & $\mathrm{n}=27$ & & & & & $\begin{array}{l}\text { Depression } \\
\text { Quality of life* }\end{array}$ \\
\hline \multirow[t]{2}{*}{ Han (2016) [24] } & \multirow[t]{2}{*}{ Republic of Korea } & \multirow{2}{*}{$\begin{array}{l}\text { Non-equivalent } \\
\text { control } \\
\text { group design }\end{array}$} & \multicolumn{2}{|c|}{$\begin{array}{l}\text { Full-time employees from } \\
\text { a public organization (61) }\end{array}$} & \multirow[t]{2}{*}{ 2-day forest therapy camp ${ }^{+}$} & \multirow[t]{2}{*}{$\begin{array}{l}\text { Normal } \\
\text { daily routines }\end{array}$} & \multirow{2}{*}{$\begin{array}{l}\text { BDI } \\
\text { The Euro Qol Visual } \\
\text { Analog Scale (EQ-VAS) }\end{array}$} & \multirow{2}{*}{$\begin{array}{l}\text { Heart Rate Variability } \\
\text { (HRV) } \\
\text { Natural Killer Cell } \\
\text { (NK cell) }\end{array}$} & $\begin{array}{l}\text { Self-rated health condition } \\
\text { Depression* } \\
\text { Ani EQ-VAS* }\end{array}$ \\
\hline & & & $\mathrm{n}=33$ & $\mathrm{n}=28$ & & & & & $\begin{array}{l}\text { HRV } \\
\text { NK cell * }\end{array}$ \\
\hline \multirow{3}{*}{$\begin{array}{l}\text { Horiuchi } \\
\text { (2014) [25] }\end{array}$} & \multirow{3}{*}{ Japan } & \multirow{3}{*}{ Crossover trial } & \multicolumn{2}{|c|}{ Healthy adults $(\mathrm{n}=15)$} & \multirow{3}{*}{$\begin{array}{l}\text { Viewing the forest while seated on } \\
\text { a comfortable chair for } 15 \mathrm{~min}- \\
\text { Switched sites with } 30 \mathrm{~min} \text { interval }\end{array}$} & \multirow{3}{*}{$\begin{array}{l}\text { Conducted } \\
\text { same activity } \\
\text { in an enclosed } \\
\text { condition }\end{array}$} & \multirow{3}{*}{$\begin{array}{l}\text { Profile of Mood States } \\
\text { (POMS) }\end{array}$} & \multirow{3}{*}{$\begin{array}{l}\text { BP } \\
\text { HRV } \\
\text { Salivary Amylase } \\
\text { (sAMY) }\end{array}$} & $\begin{array}{l}\text { Tension-anxiety * } \\
\text { Depression * }\end{array}$ \\
\hline & & & \multirow{2}{*}{\multicolumn{2}{|c|}{$\mathrm{n}=15$}} & & & & & $\begin{array}{l}\text { Anger-hostility } \\
\text { Fatigue** } \\
\text { Confusion* } \\
\text { Vigor }\end{array}$ \\
\hline & & & & & & & & & $\begin{array}{l}\mathrm{BP}^{*} \\
\mathrm{HR} * \mathrm{HF} *, \mathrm{LF} / \mathrm{HF} * \\
\text { Salivary Amylase }\end{array}$ \\
\hline \multirow{3}{*}{ Ji (2012) [26] } & & & $\begin{array}{l}\text { Healthy } \\
(\mathrm{n}=12)\end{array}$ & e adult & & & & & $\begin{array}{l}\text { Tension-Anxiety * } \\
\text { Depression * }\end{array}$ \\
\hline & Republic of Korea & Crossover trial & $\mathrm{n}=12$ & & $\begin{array}{l}\text { Viewing }(15 \mathrm{~min}) \text { and walking } \\
(25 \mathrm{~min}) \text { in the forest-Switched } \\
\text { sites with } 24 \mathrm{~h} \text {. interval }\end{array}$ & $\begin{array}{l}\text { Conducted same } \\
\text { activities in } \\
\text { the urban }\end{array}$ & POMS & $\begin{array}{l}\text { BP } \\
\text { HR } \\
\text { Amylase }\end{array}$ & $\begin{array}{l}\text { Anger-hostility * } \\
\text { Fatigue * } \\
\text { Confusion * } \\
\text { Vigor }\end{array}$ \\
\hline & & & & & & & & & $\begin{array}{l}\mathrm{BP}^{*} \\
\mathrm{HR} * \\
\text { Amylase concentration * }\end{array}$ \\
\hline
\end{tabular}


Table 2. Cont.

\begin{tabular}{|c|c|c|c|c|c|c|c|c|c|}
\hline \multirow{3}{*}{ Authors (Year) } & \multirow{3}{*}{ Country } & \multirow{3}{*}{ Research Design } & \multicolumn{2}{|c|}{ Participants (N) } & \multirow{3}{*}{ Intervention(s) } & \multirow{3}{*}{ Control } & \multirow{2}{*}{\multicolumn{2}{|c|}{ Measurements }} & \multirow{3}{*}{ Outcomes } \\
\hline & & & \multirow{2}{*}{ Exp. } & \multirow{2}{*}{ Cont. } & & & & & \\
\hline & & & & & & & Self-Report Measures & Physiological Measures & \\
\hline \multirow[b]{2}{*}{ Kim (2012) [27] } & \multirow[b]{2}{*}{ Republic of Korea } & \multirow[b]{2}{*}{ Crossover trial } & \multicolumn{2}{|c|}{ Healthy students $(n=50)$} & \multirow{2}{*}{$\begin{array}{l}\text { Forest healing program-Viewing } \\
\text { ( } 15 \mathrm{~min}) \text { and walking }(15 \mathrm{~min}) \text { in a forest } \\
\text { park landscape- }-\mathrm{Switched} \text { sites } \\
\text { with } 24 \mathrm{~h} \text {. interval }\end{array}$} & \multirow{2}{*}{$\begin{array}{l}\text { Conducted same } \\
\text { activities in the } \\
\text { urban forest }\end{array}$} & \multirow{2}{*}{$\begin{array}{l}\text { POMS } \\
\text { Semantic Differential } \\
\text { (SD) method }\end{array}$} & \multirow[b]{2}{*}{ None } & \multirow{2}{*}{$\begin{array}{l}\text { Tension-anxiety } \\
\text { Depression } \\
\text { Anger-hostility * } \\
\text { Fatigue } \\
\text { Confusion * } \\
\text { Vigor * } \\
\text { Total moods disturbance * } \\
\text { Emotion (Pleasant *, Natural *) }\end{array}$} \\
\hline & & & \multicolumn{2}{|l|}{$\mathrm{n}=50$} & & & & & \\
\hline \multirow{3}{*}{ Lee (2011) [28] } & \multirow{3}{*}{ Japan } & \multirow{3}{*}{ Crossover trial } & \multicolumn{2}{|c|}{ Healthy students $(n=12)$} & \multirow{3}{*}{$\begin{array}{l}\text { Forest bathing-Viewing the forest }(15 \\
\text { min)-Switched sites with } 24 \mathrm{~h} \text {. interval }\end{array}$} & \multirow{3}{*}{$\begin{array}{l}\text { Conducted same } \\
\text { activity in the } \\
\text { urban }\end{array}$} & \multirow{3}{*}{$\begin{array}{l}\text { POMS } \\
\text { SD method }\end{array}$} & \multirow{3}{*}{$\begin{array}{l}\text { HRV } \\
\text { Salivary Cortisol } \\
\text { Pulse Rate (PR) } \\
\text { BP }\end{array}$} & $\begin{array}{l}\text { Emotion }{ }^{*} \\
\text { Tension-anxiety* }\end{array}$ \\
\hline & & & \multirow{2}{*}{\multicolumn{2}{|c|}{$\mathrm{n}=12$}} & & & & & $\begin{array}{l}\text { Depression } \\
\text { Anger-hostility } \\
\text { Fatigue * } \\
\text { Confusion * } \\
\text { Vigor * } \\
\text { Total moods disturbance * }\end{array}$ \\
\hline & & & & & & & & & $\begin{array}{l}\text { Parasympathetic nervous } \\
\text { activity* } \\
\text { Sympathetic activity * } \\
\text { Salivary cortisol * } \\
\text { PR * }\end{array}$ \\
\hline \multirow{3}{*}{ Lee (2014) [29] } & \multirow{3}{*}{ Japan } & \multirow{3}{*}{ Crossover trial } & \multicolumn{2}{|c|}{ Healthy students $(\mathrm{n}=48)$} & \multirow{3}{*}{$\begin{array}{l}\text { Forest therapy program-Forest walking } \\
(12-15 \mathrm{~min}) \text {, self-paced walking in the } \\
\text { forest environments-Switched sites with } \\
24 \mathrm{~h} \text {. interval }\end{array}$} & \multirow{3}{*}{$\begin{array}{l}\text { Conducted same } \\
\text { activities in the } \\
\text { urban }\end{array}$} & \multirow{3}{*}{$\begin{array}{l}\text { SD method } \\
\text { The feeling of refreshed } \\
\text { questionnaire } \\
\text { POMS } \\
\text { The Spielberger } \\
\text { State-Trait Anxiety } \\
\text { Inventory (STAI) } \\
\text { questionnaire }\end{array}$} & & $\begin{array}{l}\text { Emotion* } \\
\text { Tension-anxiety* }\end{array}$ \\
\hline & & & \multirow{2}{*}{\multicolumn{2}{|c|}{$\mathrm{n}=48$}} & & & & $\begin{array}{l}\mathrm{HRV} \\
\mathrm{BP}\end{array}$ & $\begin{array}{l}\text { Depression } \\
\text { Anger-hostility * } \\
\text { Fatigue ** } \\
\text { Confusion * } \\
\text { Vigor * } \\
\text { State anxiety level * }\end{array}$ \\
\hline & & & & & & & & & $\begin{array}{l}\ln (\mathrm{LF} / \mathrm{HF})^{*} \\
\mathrm{HR} * \\
\mathrm{BP}\end{array}$ \\
\hline \multirow[b]{2}{*}{ Lim (2014) [30] } & & Non-equivalent & $\begin{array}{l}\text { Senior cit } \\
\text { a nursing }\end{array}$ & me in $(\mathrm{n}=64)$ & Forest therapy program—- & $\begin{array}{l}\text { Cont. 1: Indoor } \\
\text { therapy }\end{array}$ & & & \\
\hline & Republic of Korea & $\begin{array}{l}\text { control group } \\
\text { design }\end{array}$ & $\mathrm{n}=22$ & $\begin{array}{l}\text { Cont. } 1 \\
\mathrm{n}=21 \\
\text { Cont. } 2 \\
\mathrm{n}=21\end{array}$ & $\begin{array}{l}\text { Once a week (for } 90 \mathrm{~min} \text { ) for } \\
11 \text { weeks-Meditation- } \\
\text { Experiencing forest }\end{array}$ & $\begin{array}{l}\text { (Conducted same } \\
\text { activities program } \\
\text { in the room) } \\
\text { Cont. 2: Normal } \\
\text { daily routines }\end{array}$ & $\begin{array}{l}\text { Self-esteem } \\
\text { Depression }\end{array}$ & None & $\begin{array}{l}\text { Self-esteem * } \\
\text { Depression * }\end{array}$ \\
\hline
\end{tabular}


Table 2. Cont.

\begin{tabular}{|c|c|c|c|c|c|c|c|c|c|}
\hline \multirow{2}{*}{ Authors (Year) } & \multirow{2}{*}{ Country } & \multirow{2}{*}{ Research Design } & \multicolumn{2}{|c|}{ Participants (N) } & \multirow{2}{*}{ Intervention(s) } & \multirow{2}{*}{ Control } & \multirow{2}{*}{\multicolumn{2}{|c|}{ Measurements }} & \multirow{2}{*}{ Outcomes } \\
\hline & & & Exp. & Cont. & & & & & \\
\hline \multirow{3}{*}{$\begin{array}{l}\text { Mao G.X. } \\
\text { (2012) [19] }\end{array}$} & \multirow{3}{*}{ China } & \multirow{3}{*}{ RCT } & \multicolumn{2}{|c|}{ Healthy students $(n=20)$} & \multirow{3}{*}{$\begin{array}{l}\text { Forest bathing-Twice a day for two } \\
\text { days-Walking in the forest area (for } 90 \\
\text { min), with a } 10 \text {-min rest during the walk }\end{array}$} & \multirow{3}{*}{$\begin{array}{l}\text { Conducted same } \\
\text { activities in the } \\
\text { city area }\end{array}$} & \multirow{3}{*}{ POMS } & \multirow{3}{*}{$\begin{array}{l}\text { BP } \\
\text { Cytokine } \\
\text { Enzyme-linked } \\
\text { immunoassay }\end{array}$} & \multirow{3}{*}{$\begin{array}{l}\text { Tension-anxiety * } \\
\text { Depression-dejection * } \\
\text { Anger-hostility * } \\
\text { Fatigue-inertia * } \\
\text { Confusion-bewilderment } \\
\text { Vigor-activity * } \\
\text { BP * } \\
\text { Cytokine: IL-6 * } \\
\text { Enzyme-linked immunoassay: } \\
\text { Renin *, AGT * }\end{array}$} \\
\hline & & & \multirow[t]{2}{*}{$\mathrm{n}=10$} & \multirow[t]{2}{*}{$\mathrm{n}=10$} & & & & & \\
\hline & & & & & & & & & \\
\hline \multirow{2}{*}{ Shin (1996) [31] } & \multirow{2}{*}{ Republic of Korea } & \multirow{2}{*}{$\begin{array}{l}\text { Non-equivalent } \\
\text { control group } \\
\text { design }\end{array}$} & \multicolumn{2}{|c|}{$\begin{array}{l}\text { Students (BDI scores: } \\
18-30)(\mathrm{n}=64)\end{array}$} & \multirow{2}{*}{$\begin{array}{l}\text { 5-day forest program-Group } \\
\text { presentation, team exercise, hiking, and } \\
\text { climbing the mountain }\end{array}$} & \multirow{2}{*}{$\begin{array}{l}\text { Normal } \\
\text { daily routines }\end{array}$} & \multirow{2}{*}{ BDI } & \multirow{2}{*}{ None } & \multirow{2}{*}{ Depression * } \\
\hline & & & $\mathrm{n}=32$ & $\mathrm{n}=32$ & & & & & \\
\hline \multirow[b]{2}{*}{ Song (2011) [32] } & \multirow[b]{2}{*}{ Japan } & \multirow[b]{2}{*}{ Crossover trial } & \multicolumn{2}{|c|}{$\begin{array}{l}\text { Healthy male adults } \\
(\mathrm{n}=18)\end{array}$} & \multirow[b]{2}{*}{$\begin{array}{l}\text { Walking in the urban forest }(20 \\
\text { min)-Switched sites with } 24 \mathrm{~h} \text {. interval }\end{array}$} & \multirow[b]{2}{*}{$\begin{array}{l}\text { Conducted same } \\
\text { activity in the } \\
\text { urban area }\end{array}$} & \multirow[b]{2}{*}{$\begin{array}{l}\text { POMS } \\
\text { STAI } \\
\text { The Symptom Checklist } \\
\text { (SCL-90) } \\
\text { Type A behavior }\end{array}$} & & $\begin{array}{l}\text { Tension-anxiety } \\
\text { Depression * }\end{array}$ \\
\hline & & & $\mathrm{n}=18$ & & & & & None & $\begin{array}{l}\text { Anger-hostility * } \\
\text { Fatigue * } \\
\text { Confusion } \\
\text { Vigor * } \\
\text { Total mood disturbance * } \\
\text { State-anxiety * } \\
\text { Hostility * } \\
\text { Anxiety * } \\
\text { Obsessive-compulsive * } \\
\text { Somatization * }\end{array}$ \\
\hline & & & $\begin{array}{l}\text { Female } n \\
\text { students }\end{array}$ & $\begin{array}{l}\text { ing college } \\
=53 \text { ) }\end{array}$ & & & & & $\begin{array}{l}\text { Tension* } \\
\text { Attack* }\end{array}$ \\
\hline Song (2014) [33] & Republic of Korea & $\begin{array}{l}\text { Non-equivalent } \\
\text { control } \\
\text { groupdesign }\end{array}$ & $\mathrm{n}=27$ & $\mathrm{n}=26$ & $\begin{array}{l}\text { Forest Healing Program-Once a week } \\
\text { (for } 3 \mathrm{~h} \text { ) for } 12 \text { weeks-Forest } \\
\text { meditation-Natural healing play-Stress } \\
\text { management }\end{array}$ & $\begin{array}{l}\text { Normal daily } \\
\text { routines }\end{array}$ & $\begin{array}{l}\text { Stress Response } \\
\text { The Spiritual } \\
\text { Assessment Scale }\end{array}$ & None & $\begin{array}{l}\text { Somatization } \\
\text { Anger } \\
\text { Depression } \\
\text { Fatigue } \\
\text { Frustration * } \\
\text { Total points of stress responses * } \\
\text { Total points of spirituality * }\end{array}$ \\
\hline & & & Healthy & dents $(n=11)$ & & & & & Emotion (Comfortable, Relaxed \\
\hline Song (2015) [23] & Japan & Crossover trial & $\mathrm{n}=11$ & & $\begin{array}{l}\text { Walking in the forest in the morning (for } \\
15 \mathrm{~min} \text { ) Viewing the forest (for } 15 \mathrm{~min} \text { ) in } \\
\text { the afternoon-Switched sites with } 24 \mathrm{~h} \text {. } \\
\text { interval }\end{array}$ & $\begin{array}{l}\text { Conducted same } \\
\text { activities in the } \\
\text { urban area }\end{array}$ & $\begin{array}{l}\text { SD method } \\
\text { An inventory for the } \\
\text { measurement of } \\
\text { self-reported stress and } \\
\text { arousal } \\
\text { POMS } \\
\text { STAI }\end{array}$ & HRV & $\begin{array}{l}\text { Feeling refreshed *) } \\
\text { Tension-anxiety * } \\
\text { Depression * } \\
\text { Anger-hostility } \\
\text { Fatigue * } \\
\text { Confusion } \\
\text { Vigor } \\
\text { Anxiety } \\
\text { * }\end{array}$ \\
\hline & & & & & & & & & $\begin{array}{l}\text { Overall mean } \ln (\mathrm{HF})^{*} \\
\text { Overall mean } \mathrm{HR}^{*}\end{array}$ \\
\hline
\end{tabular}


Table 2. Cont.

\begin{tabular}{|c|c|c|c|c|c|c|c|c|c|}
\hline \multirow{3}{*}{ Authors (Year) } & \multirow{3}{*}{ Country } & \multirow{3}{*}{ Research Design } & \multicolumn{2}{|c|}{ Participants (N) } & \multirow{3}{*}{ Intervention(s) } & \multirow{3}{*}{ Control } & \multirow{2}{*}{\multicolumn{2}{|c|}{ Measurements }} & \multirow{3}{*}{ Outcomes } \\
\hline & & & \multirow{2}{*}{ Exp. } & \multirow{2}{*}{ Cont. } & & & & & \\
\hline & & & & & & & Self-Report Measures & Physiological Measures & \\
\hline \multirow[b]{2}{*}{$\begin{array}{l}\text { Takayama } \\
\text { (2014) [34] }\end{array}$} & \multirow[b]{2}{*}{ Japan } & \multirow[b]{2}{*}{ Crossover trial } & \multicolumn{2}{|c|}{ Healthy students $(n=45)$} & \multirow[b]{2}{*}{$\begin{array}{l}\text { Forest bathing-Walking in the forest in } \\
\text { morning }(15 \mathrm{~min}) \text {-Viewing the forest in } \\
\text { the afternoon }(15 \mathrm{~min}) \text {-Switched sites } \\
\text { with } 24 \mathrm{~h} \text {. interval }\end{array}$} & \multirow[b]{2}{*}{$\begin{array}{l}\text { Conducted same } \\
\text { activities in the } \\
\text { urban area }\end{array}$} & \multirow{2}{*}{$\begin{array}{l}\text { POMS } \\
\text { Positive and Negative } \\
\text { Affect Schedule } \\
\text { (PANAS) } \\
\text { The Restorative } \\
\text { Outcome Scale (ROS) } \\
\text { The Subjective Vitality } \\
\text { Scale }\end{array}$} & \multirow[b]{2}{*}{ None } & \multirow[b]{2}{*}{$\begin{array}{l}\text { Tension-anxiety * } \\
\text { Depression * } \\
\text { Anger-hostility } \\
\text { Fatigue } \\
\text { Confusion * } \\
\text { Vigor * } \\
\text { Negative affect* * } \\
\text { Positive Affect* } \\
\text { The ROS score * } \\
\text { The total SVS score * }\end{array}$} \\
\hline & & & \multicolumn{2}{|l|}{$\mathrm{n}=45$} & & & & & \\
\hline \multirow{2}{*}{ Yang (2011) [35] } & \multirow{2}{*}{ Republic of Korea } & \multirow{2}{*}{$\begin{array}{l}\text { Non-equivalent } \\
\text { control } \\
\text { groupdesign }\end{array}$} & \multicolumn{2}{|c|}{$\begin{array}{l}\text { Alcoholics' families } \\
(\mathrm{n}=46)\end{array}$} & \multirow{2}{*}{$\begin{array}{l}\text { 6-day forest program-Forest } \\
\text { experience-Drawing a forest-Day and } \\
\text { Night walk }\end{array}$} & \multirow{2}{*}{$\begin{array}{l}\text { Normal daily } \\
\text { Routines }\end{array}$} & \multirow{2}{*}{$\begin{array}{l}\text { Spiritual health } \\
\text { Inventory (SHI) } \\
\text { BDI } \\
\text { The Rosenberg } \\
\text { Self-Esteem Scale }\end{array}$} & \multirow[b]{2}{*}{ None } & \multirow{2}{*}{$\begin{array}{l}\text { Spiritual health * } \\
\text { Depression * } \\
\text { Self-esteem * }\end{array}$} \\
\hline & & & $\mathrm{n}=24$ & $\mathrm{n}=22$ & & & & & \\
\hline \multirow[b]{2}{*}{ You (2014) [36] } & \multirow[b]{2}{*}{ Republic of Korea } & \multirow{2}{*}{$\begin{array}{l}\text { Non-equivalent } \\
\text { control group } \\
\text { design }\end{array}$} & \multicolumn{2}{|c|}{ Healthy females $(\mathrm{n}=20)$} & \multirow{2}{*}{$\begin{array}{l}\text { Sallimyok (Forest Therapy) } \\
\text { Meditation } \\
\text { Walking } \\
\text { Qi-Qong program }\end{array}$} & \multirow[b]{2}{*}{$\begin{array}{l}\text { Normal daily } \\
\text { routines }\end{array}$} & \multirow{2}{*}{$\begin{array}{l}\text { Zung Self-Rating } \\
\text { Depression Scale } \\
\text { Psychological } \\
\text { Well-Being Scale }\end{array}$} & \multirow[b]{2}{*}{ None } & \multirow{2}{*}{$\begin{array}{l}\text { Depression * } \\
\text { Psychological well-being * }\end{array}$} \\
\hline & & & $\mathrm{n}=10$ & $\mathrm{n}=10$ & & & & & \\
\hline
\end{tabular}

Note: Exp.: Experimental group, Cont.: Control group; ${ }^{*}$ Significant finding; 2 -day forest therapy camp; ${ }^{\dagger}$ consisted of walking, therapeutic activities, psychoeducation for coping with pain and stress, bodily exercises and mindfulness-based meditation in the forest and indoor music therapy. 
Table 3. Summary of Studies for Adults with Health Problems ( $=12)$.

\begin{tabular}{|c|c|c|c|c|c|c|c|c|c|}
\hline \multirow{2}{*}{ Authors (Year) } & \multirow{2}{*}{ Country } & \multirow{2}{*}{ Research Design } & \multicolumn{2}{|c|}{ Participants (N) } & \multirow{2}{*}{ Intervention } & \multirow{2}{*}{ Control } & \multicolumn{2}{|c|}{ Measurement } & \multirow{2}{*}{ Outcome } \\
\hline & & & Exp. & Cont. & & & Self-Report Measures & Physiological Measures & \\
\hline \multirow{2}{*}{ Barton (2012) [12] } & \multirow{2}{*}{ UK } & \multirow{2}{*}{$\begin{array}{l}\text { Non-equivalent } \\
\text { control group design }\end{array}$} & \multicolumn{2}{|c|}{$\begin{array}{l}\text { Adults with a mental health } \\
\text { problem }(\mathrm{n}=53)\end{array}$} & \multirow{2}{*}{$\begin{array}{l}\text { Green exercise } \\
\text { Walking in the green spaces ( } 45 \\
\text { min) }\end{array}$} & \multirow{2}{*}{$\begin{array}{l}\text { Cont. 1: Swimming } \\
\text { Cont. 2: Social activities }\end{array}$} & \multirow{2}{*}{$\begin{array}{l}\text { The Rosenberg } \\
\text { Self-Esteem Scale } \\
\text { POMS }\end{array}$} & \multirow{2}{*}{ None } & \multirow{2}{*}{$\begin{array}{l}\text { Self-esteem * } \\
\text { Overall mood }\end{array}$} \\
\hline & & & $\mathrm{n}=24$ & $\begin{array}{l}\text { Cont. } 1(\mathrm{n}=14) \\
\text { Cont. } 2(\mathrm{n}=15)\end{array}$ & & & & & \\
\hline \multirow[b]{2}{*}{ Choi (2014) [37] } & \multirow[b]{2}{*}{ Republic of Korea } & \multirow{2}{*}{$\begin{array}{l}\text { Non-equivalent } \\
\text { control group design }\end{array}$} & \multicolumn{2}{|c|}{ Cancer patients $(\mathrm{n}=53)$} & \multirow{2}{*}{$\begin{array}{l}\text { Forest-experience-integration } \\
\text { intervention }{ }^{\dagger} \text {-Once a week (for } \\
120 \mathrm{~min} \text { ) for } 8 \text { weeks }\end{array}$} & \multirow[b]{2}{*}{ Normal daily routines } & \multirow{2}{*}{$\begin{array}{l}\text { Zung Self-Rating } \\
\text { Depression Scale } \\
\text { Self-regulation } \\
\text { Resilience }\end{array}$} & \multirow[b]{2}{*}{ None } & \multirow{2}{*}{$\begin{array}{l}\text { Depression * } \\
\text { Resilience * }\end{array}$} \\
\hline & & & $\mathrm{n}=26$ & $\mathrm{n}=27$ & & & & & \\
\hline \multirow[b]{2}{*}{ Chun (2016) [20] } & \multirow[b]{2}{*}{ Republic of Korea } & \multirow[b]{2}{*}{ RCT } & \multicolumn{2}{|c|}{ Chronic stroke patients $(\mathrm{n}=59)$} & \multirow{2}{*}{$\begin{array}{l}\text { 4-day forest therapy } \\
\text { program-Meditation, } \\
\text { Experiencing the forest through } \\
\text { all five senses-Walking in } \\
\text { the forest }\end{array}$} & \multirow{2}{*}{$\begin{array}{l}\text { Stayed in a hotel and } \\
\text { participated in } \\
\text { meditation and walking } \\
\text { activities }\end{array}$} & \multirow{2}{*}{$\begin{array}{l}\text { BDI } \\
\text { Hamilton Depression } \\
\text { rating scale (HAM-D17) } \\
\text { STAI }\end{array}$} & \multirow[b]{2}{*}{ None } & \multirow{2}{*}{$\begin{array}{l}\text { Depression * } \\
\text { Anxiety * }\end{array}$} \\
\hline & & & $\mathrm{n}=30$ & $\mathrm{n}=29$ & & & & & \\
\hline & & & Psychiatr & atients $(\mathrm{n}=16)$ & & & $\begin{array}{l}\text { The Instrument of } \\
\text { Oriental Medical }\end{array}$ & & $\begin{array}{l}\text { Hwa-Byung symptoms* } \\
\text { Depression * }\end{array}$ \\
\hline Hong (2012) [13] & Republic of Korea & Crossover trial & $\mathrm{n}=16$ & & $\begin{array}{l}\text { 3-day forests healing program } \\
\text { ¥for patients with }\end{array}$ & $\begin{array}{l}\text { Conducted regular } \\
\text { diet and exercise }\end{array}$ & $\begin{array}{l}\text { Evaluation for } \\
\text { Hwa-Byung BDI } \\
\text { STAI } \\
\text { State-Trait Anger }\end{array}$ & Heart Rate Variability & $\begin{array}{l}\text { Anxiety* } \\
\text { State anger* } \\
\text { Trait anger } \\
\text { Quality of Life* } \\
\end{array}$ \\
\hline & & & & & $\begin{array}{l}\text { Hwa--Byung-Washout period } \\
\text { (28 days) }\end{array}$ & $\begin{array}{l}\text { program ( } 3 \text { times } \\
\text { a day) in the forest }\end{array}$ & $\begin{array}{l}\text { Expression Inventory } \\
\text { (STAXI) } \\
\text { WHO Quality of Life } \\
\text { Scale Abbreviated } \\
\text { Version } \\
\text { (WHOQOL-BREF) }\end{array}$ & & $\begin{array}{l}\text { Mean } \mathrm{HR}^{*} \\
\text { Heart rate variability } \\
\text { LF/HF }\end{array}$ \\
\hline & & & Psychiatr & atients $(n=15)$ & 3-day forests healing programs $\ddagger$ & Conducted regular & & & Depression * \\
\hline Hong (2013) [14] & Republic of Korea & Crossover trial & $\mathrm{n}=15$ & & $\begin{array}{l}\text { for cognitive } \\
\text { improvement-Washout period } \\
\text { (28 days) }\end{array}$ & $\begin{array}{l}\text { diet and exercise } \\
\text { program ( } 3 \text { times } \\
\text { a day) in the forest }\end{array}$ & $\begin{array}{l}\text { STAI } \\
\text { STAXI } \\
\text { WHOQOL-BREF }\end{array}$ & None & $\begin{array}{l}\text { Anger } \\
\text { Quality of Life* }\end{array}$ \\
\hline & & & COPD pa & $\mathrm{z}=20$ ) & & & & & $\begin{array}{l}\text { Tension-anxiety * } \\
\text { Depression-dejection * }\end{array}$ \\
\hline Jia (2016) [21] & China & RCT & $\mathrm{n}=10$ & $\mathrm{n}=10$ & $\begin{array}{l}\text { One-day forest bathing } \\
\text { trip-Forest walking } \\
\text { in the morning (for } 90 \mathrm{~min} \text { ) and } \\
\text { in the afternoon (for } 90 \mathrm{~min} \text { ) }\end{array}$ & $\begin{array}{l}\text { One-day trip for } \\
\text { urban walking }\end{array}$ & POMS & $\begin{array}{l}\text { Cortisol } \\
\text { Epinephrine }\end{array}$ & $\begin{array}{l}\text { Anger-hostility }{ }^{*} \\
\text { Fatigue-inertia } \\
\text { Confusion-bewilderment } \\
\text { Vigor-activity }\end{array}$ \\
\hline & & & & & & & & & $\begin{array}{l}\text { Cortisol* } \\
\text { Epinephrine* }\end{array}$ \\
\hline & & & Psychiatr & tients $(\mathrm{n}=20)$ & & & & & $\begin{array}{l}\text { Tension-anxiety } \\
\text { Depression }\end{array}$ \\
\hline Kim (2015) [15] & Republic of Korea & $\begin{array}{l}\text { Non-equivalent } \\
\text { control group design }\end{array}$ & $\mathrm{n}=10$ & $\mathrm{n}=10$ & $\begin{array}{l}\text { Forest experience program-5 } 5 \\
\text { times in } 2 \text { weeks }(60 \mathrm{~min} \text { each } \\
\text { time)-Handkerchief } \\
\text { dyeing-Decorating a frame } \\
\text { using natural items }\end{array}$ & TAU & $\begin{array}{l}\text { Korean Version of } \\
\text { Profile of Mood } \\
\text { State-Brief (K-POMS-B) } \\
\text { BDI }\end{array}$ & Salivary cortisol & $\begin{array}{l}\text { Anger-hostility } \\
\text { Fatigue } \\
\text { Confusion } \\
\text { Vigor } \\
\text { Total moods } \\
\text { disturbance }\end{array}$ \\
\hline & & & & & & & & & Cortisol $^{*}$ \\
\hline
\end{tabular}


Table 3. Cont

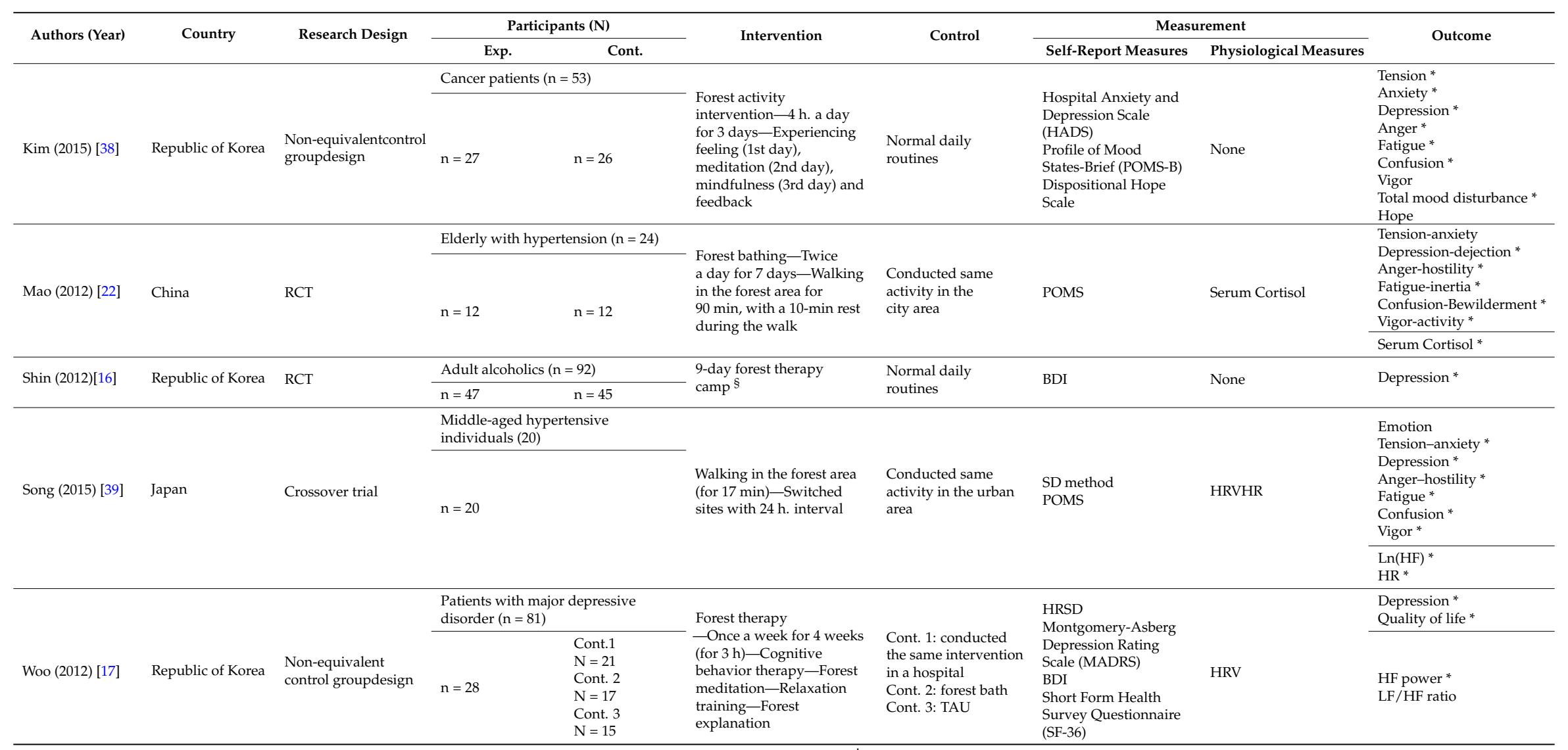

Note. Exp.: Experimental group, Cont.: Control group; TAU: Treatment-as-usual; ${ }^{*}$ Significant finding; ${ }^{\dagger}$ three-day forest-experience-integration intervention consisted of preparation phase (30 $\mathrm{min})$, physical intervention (20 min), psychological intervention $(20 \mathrm{~min})$, physical intervention $(20 \mathrm{~min})$, and completion phase (30 min).; $;$ three-day forests healing program conducted in the healing forest area and consisted of forest healing activities and oriental medicine treatments. Forest healing activities included various activities in the forest, such as exercise, Qi-Qong program, and experiencing forest using five senses. Oriental medicine treatments included natural herbal diet, herbal footbath therapy, aroma therapy, herbal tea therapy, and oriental medicine music; \& nine-day forest therapy camp consisted of three sessions and each session lasted for three days. Each session included various therapeutic activities including nature games and nature interpretation (1st session); mountain-climbing, trekking, and orienteering (2nd session); nature-meditation and counseling in forest environment (3rd session). 


\subsubsection{Format and Content of Forest Therapy}

The forest therapy programs tested in these 28 studies varied in terms of format and content of the programs. The length of time that the interventions were undertaken ranged from one day to twelve weeks. The duration of the forest therapy ranged from twelve minutes [29] to three hours [33]. About one third of the studies [23,26-29,32,34,39] offered forest therapy programs and control conditions (such as activities in downtown) every other day during the two-day periods. Three studies $[12,21,25]$ used a one-time intervention that lasted a few hours to half a day. One study did not report duration details of the intervention [36].

Regarding the content of forest therapy, walking in the forest was the key component of the forest therapy that was included in most studies except one [28]. Other therapeutic activities included in forest therapy programs were experiencing forest through the five senses (seeing, hearing, touching, smelling, and tasting), forest viewing, forest meditation, Qi-Qong, aromatherapy, herbal tea therapy, and craftwork using natural materials.

\subsubsection{Depression Measures}

The most commonly used self-report measure for depression in these 28 studies was the Profile of Mood States (POMS). For articles published in English, nine studies $[12,19,21,22,25,28,29,34,39]$ used POMS to assess the level of depression and three studies [28,29,39] used Semantic differential (SD) method. Other scales used by the studies included the Hamilton Rating Scales for Depression [20], Beck Depression Inventory (BDI) $[16,20,24,31]$, positive and negative affect schedule (PANAS) [34]. For studies published in Korean, the POMS [15,23,26,27,32,38] and BDI $[13-15,17,18,35]$ were the most commonly used scale. Other scales used to measure depression were the Hospital Anxiety and Depression Scale (HADS) [38], Hamilton Rating Scales for Depression [17], Montgomery-Asberg Depression Rating Scales [17], Symptom Check List (SCL-90-R) [32], and Zung Self-Rating Depression Scale (SDS) [36]. Fourteen studies [13,15,17-19,21-26,28,29,39] used both self-report, paper-and-pencil questionnaires, and physiological measures, while 14 studies $[12,14,16,20,27,30-38]$ used only self-report, paper-and-pencil questionnaires. Physiological or objective measures included heart rate variability (HRV), blood pressure, heart rate, and amylase concentration. Detailed information of the measures included in these studies is summarized in Tables 2 and 3.

\subsubsection{Effects of Forest Therapy on Depression}

All 28 studies assessed the level of depression before and after the intervention; however, no study conducted additional long-term follow-up assessments. Regarding the changes in the level of depression, 21 studies showed significant improvement in depression, whereas seven studies reported no significant changes in depression compared to the control group $[12,15,18,27-29,33]$. The studies that failed to demonstrate a significant improvement in the level of depression were the ones that targeted only healthy adults and the ones that conducted "viewing or walking in the forest" activities only for the intervention group.

Regarding the differential pattern of findings associated with research design, while 8 out of 11 crossover trials and 8 out of 11 non-equivalent control group design studies reported significant improvement in depression scores, five out of six RCTs reported significant results. However, the differential patterns of findings could be partly attributable to the sample characteristics of the studies; four out of six RCTs were conducted with adults with health problems.

\subsection{Quality Assessment}

Based on the SIGN checklist, ten out of the twenty-eight studies [16,18-21,23,25,30,34,37] met an "acceptable quality" rating and the rest eighteen studies were rated as low quality [12-15,17, $22,24,26-29,31-33,35,36,38,39]$. Six studies [16,18-22] used random allocations; however, no detailed description of the procedure was provided except two studies [18,20]. Five out of six RCT studies 
included in this review met the criteria for the "acceptable quality" [16,18-21], meeting all the items in the checklist except the criterion of blinding the treatment allocation to participants/researchers. One of the RCTs was rated "low" in terms of quality because the homogeneity between the experimental group and the control group at baseline was not ensured and the significant differences between the two groups had not been adequately addressed [22]. Three out of eleven crossover trials [23,25,34] were rated "acceptable" in terms of quality because they had low drop-out rates and the only difference between the experimental and control groups was the treatment under investigation. The main reasons for the "low quality" ratings were inadequate random allocation or method of concealment used. Among 11 categories in the SIGN checklist, studies were rated to have "high risk of bias" particularly for three categories: "the assignment of participants to treatment groups is randomized," "an adequate concealment method is used," and "the design keeps participants and investigators 'blind' about treatment allocation." Please see Figure 2 for risk of bias graph.

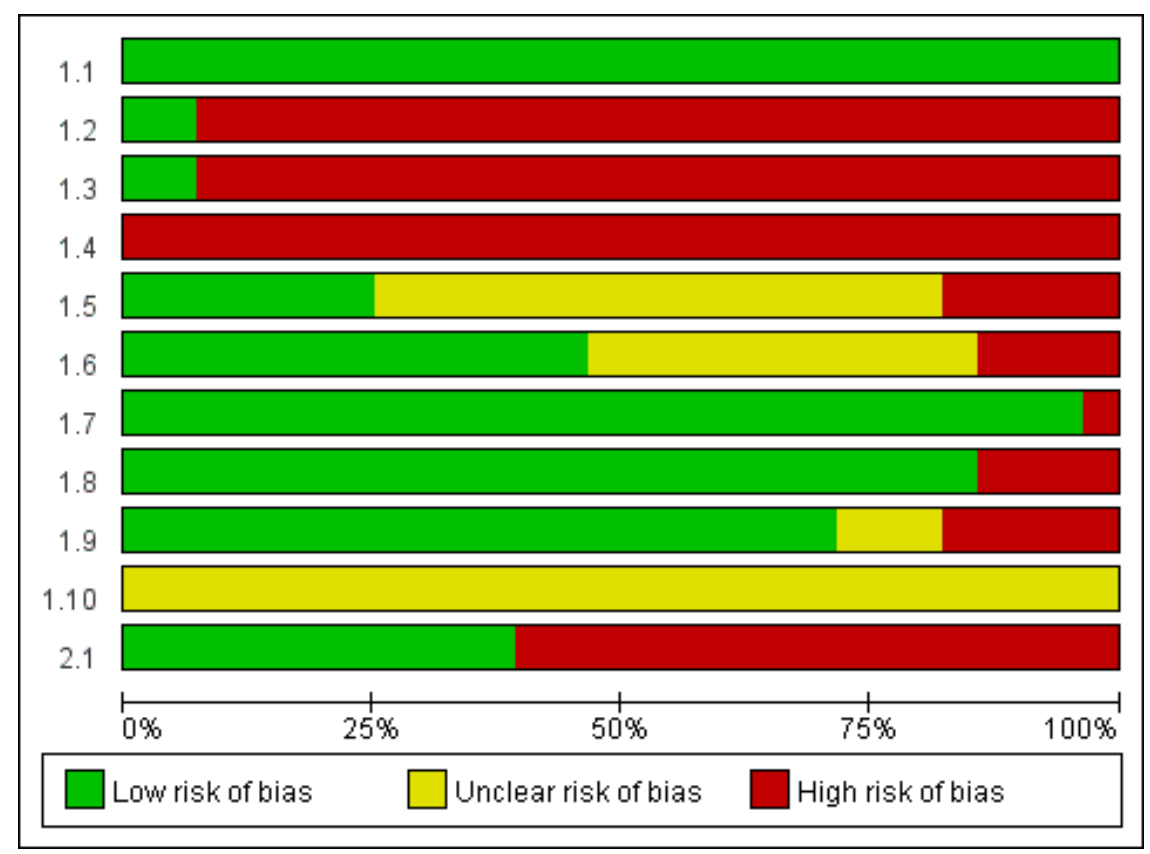

Figure 2. Risk of Bias Graph. Note: Authors' judgments about each risk of bias item presented as percentages across all included studies. 1.1 The study addresses an appropriate and clearly focused question; 1.2 The assignment of participants to treatment groups is randomized; 1.3 An adequate concealment method is used; 1.4 The design keeps participants and investigators "blind" about treatment allocation; 1.5 The treatment and control groups are similar at the start of the trial; 1.6 The only difference between groups is the treatment under investigation; 1.7 All relevant outcomes are measured in a standard, valid, and reliable way; 1.8 What percentage of the individuals or clusters recruited into each treatment arm of the study; dropped out before the study was completed? $(<20 \%=$ low risk of bias); 1.9 All the participants are analyzed in the groups that they were randomly allocated (often referred to as intention to treat analysis); 1.10 Where the study is carried out at more than one site, results are comparable for all sites; 2.1 How well was the study done to minimize bias?

\section{Discussion}

As a result of the extensive literature review, we could identify 28 studies meeting the criteria for the present review. All the studies were data-based, intervention studies with at least one comparison group. Moreover, most studies (24 out of 28 studies) were published within the last five years, confirming that forest therapy is one of the emerging therapeutic approaches and it has been gaining popularity. An analysis of the 227 regional healthcare program plans proposed in Korea between 2011 
and 2014 also revealed that 35 healthcare programs were utilizing forest resources [40]. These findings demonstrated that forest therapy is a fast-growing treatment approach used in the community.

All 28 studies varied in terms of their sample characteristics and intervention types such as format, content, and study settings. Regardless of the wide variations, in general, the studies demonstrated that forest therapy is effective in improving depression, particularly for adults with health problems. However, programs that targeted only healthy adults and the ones that used "viewing or walking in the forest" activities as the only main intervention were not effective in improving depression. Per Stigsdotter et al. forest therapy is classified into three different levels of contact: "viewing nature," "being in the presence of nearby nature," and "active participation and involvement with nature [41]." All the activities had a certain amount of health benefits [3]; however, this review revealed that "viewing nature" or "being present near nature" may not be enough to have a significant impact on the level of depression. Therefore, future studies testing the effects of forest therapy need to include a higher level or dosage of therapeutic component of forest therapy. Another possible reason for the not-significant effect of forest therapy on improving depression in healthy adults is ceiling effect [42]. For future studies, well-thought-out intervention contents and careful selection of the outcome measures and target population are recommended. It is also important to develop structured and theory-based forest therapy programs based on the scientific evidence for the specific health benefits of forests.

Despite the increasing number of studies testing the effects of the forest therapy, these published studies are still lacking methodological rigor, mainly due to a small sample size and not having an RCT design. The majority of the studies used either non-equivalent control group design or crossover design. Crossover design, or within-subjects design, increases statistical power and enables researchers to test the effect of the intervention with relatively small samples compared to studies using between-subject design. In addition, the internal validity of crossover designs is not influenced by random assignment or between-subject variation [43,44]. On the other hand, crossover design has several limitations. For crossover design, carryover effects, the treatment effect that is carried over from one experimental period to the next experimental period, needs to be carefully examined. In addition, dropout or missing data could be the significant problem because each participant serves as both the intervention and control group; therefore, the amount of contribution made by one participant is relatively large [43-46]. However, carryover effects inherited in the crossover study design were not properly addressed in the reviewed studies. Only two crossover trials conducted by the same investigator mentioned washout periods. In addition, only 4 out of 28 studies mentioned dropout rates. Overall, issues associated with dropout or missing data were not discussed in the reviewed studies.

A second issue related to methodological rigor is the inadequacy of the control group/condition employed in the non-equivalent control group design studies. The majority of the non-equivalent control group studies used "usual care" for the control group and did not properly address factors that may threaten the findings' validity such as the Rosenthal effect. In the future, well-designed studies with structurally equivalent control groups are needed to improve the quality.

Another shortcoming of these studies was the lack of reliable measures for assessing the level of depression. About half of these studies used self-report, paper-and-pencil-based questionnaires that only assessed the level of depression. Since the significant correlations between the physiological findings (e.g., electroencephalogram asymmetry) and the level of perceived depression has received attention $[47,48]$, scientists have begun to use various physiological measures to assess depression in addition to self-report questionnaires. Heart rate variability (HRV) was one of the commonly used measures in the studies included in the present paper. HRV is a physiological marker that reflects the functioning of the sympathetic and parasympathetic nervous system and is also a well-established indicator of stress and depression [49,50]. A significantly reduction in HRV has been observed among patients with depression compared to the healthy adults [51,52].

Other physiological measures that have been used to assess the level of stress and psychological conditions, including depression, natural killer cell activity [53], salivary amylase activity [54,55], salivary and serum cortisol, immunoglobulin A (IgA) concentrations [56], and urinary adrenaline 
levels [57]. In addition, electroencephalogram-based biomarkers (i.e., rACC theta, LDAEP, iAPF, P300, frontal theta activity) were found to predict the prognosis of the course of mental illness and treatment response [58]. Therefore, future studies examining the effects of forest therapy on depression need to use well-established and reliable physiological measures in addition to self-reported questionnaires to capture the full picture of the therapeutic effects of forest therapy.

Lastly, sample characteristics of the reviewed studies deserve mention. The majority of the reviewed studies targeted healthy adult participants; only three studies tested the effects of forest therapy on adults diagnosed with major depressive disorder. Therefore, the extent that the results are applicable to clinical depression is still uncertain. More studies with clinical samples are needed to establish evidence of the therapeutic value of forest therapy. Furthermore, longitudinal studies testing the long-term effects of forest therapy on depression and the changes in depressive symptoms over a span of time are needed.

A limitation of this systematic review is language bias since we only included studies that were published in English and Korean. Studies published in other languages, such as Chinese and Japanese, were not included in this review. Despite this limitation, this study increased the understanding of the therapeutic benefits of forest therapy and identified gaps in the literature.

\section{Conclusions}

This review demonstrated that forest therapy is an emerging and effective intervention for decreasing adults' depressive symptoms. However, the studies included in this review lacked methodological rigor. Future studies assessing the long-term effects of forest therapy on depression using rigorous study designs are needed.

Acknowledgments: This work has been supported by a research grant from the Korea Forest Service (No. S211214L010140).

Author Contributions: Insook Lee, Sungjae Kim, Kyung-Sook Bang, and Heeseung Choi conceived and designed the study; Heeseung Choi, Kyung-Sook Bang, MinKyung Song and Buhyun Lee conducted the systematic review and analyzed the data; Heeseung Choi, MinKyung Song, and Buhyun Lee wrote the paper; and Insook Lee, Heeseung Choi, Kyung-Sook Bang, and Sungjae Kim reviewed and refined the paper.

Conflicts of Interest: The authors declare no conflict of interest.

\section{Appendix A.}

Table A1. Search Terms Used to Identify Relevant Studies for the Review.

\begin{tabular}{|c|c|}
\hline \multicolumn{2}{|c|}{ Forest Therapy and Depression } \\
\hline \multicolumn{2}{|c|}{ Intervention } \\
\hline & Trees*/ \\
\hline & Tree/ \\
\hline & Forests/ \\
\hline & Forest/ \\
\hline & Forest Areas/ \\
\hline & Area, Forested/ \\
\hline & Areas, Forested/ \\
\hline & Forested Area/ \\
\hline 9. & Woodland/ \\
\hline & Woodlands/ \\
\hline & Forestlands / \\
\hline & Forestland/ \\
\hline & Wood/ \\
\hline 14. & Woods/ \\
\hline 15. & Shinrinyoku/ \\
\hline & Green exercise/ \\
\hline 17. & 1 OR 2 OR 3 OR 4 OR 5 OR 6 OR 7 OR 8 OR 9 OR 10 OR 11 OR 12 OR 13 OR 14 OR 15 OR 16 OR 17 \\
\hline
\end{tabular}


Table A1. Cont.

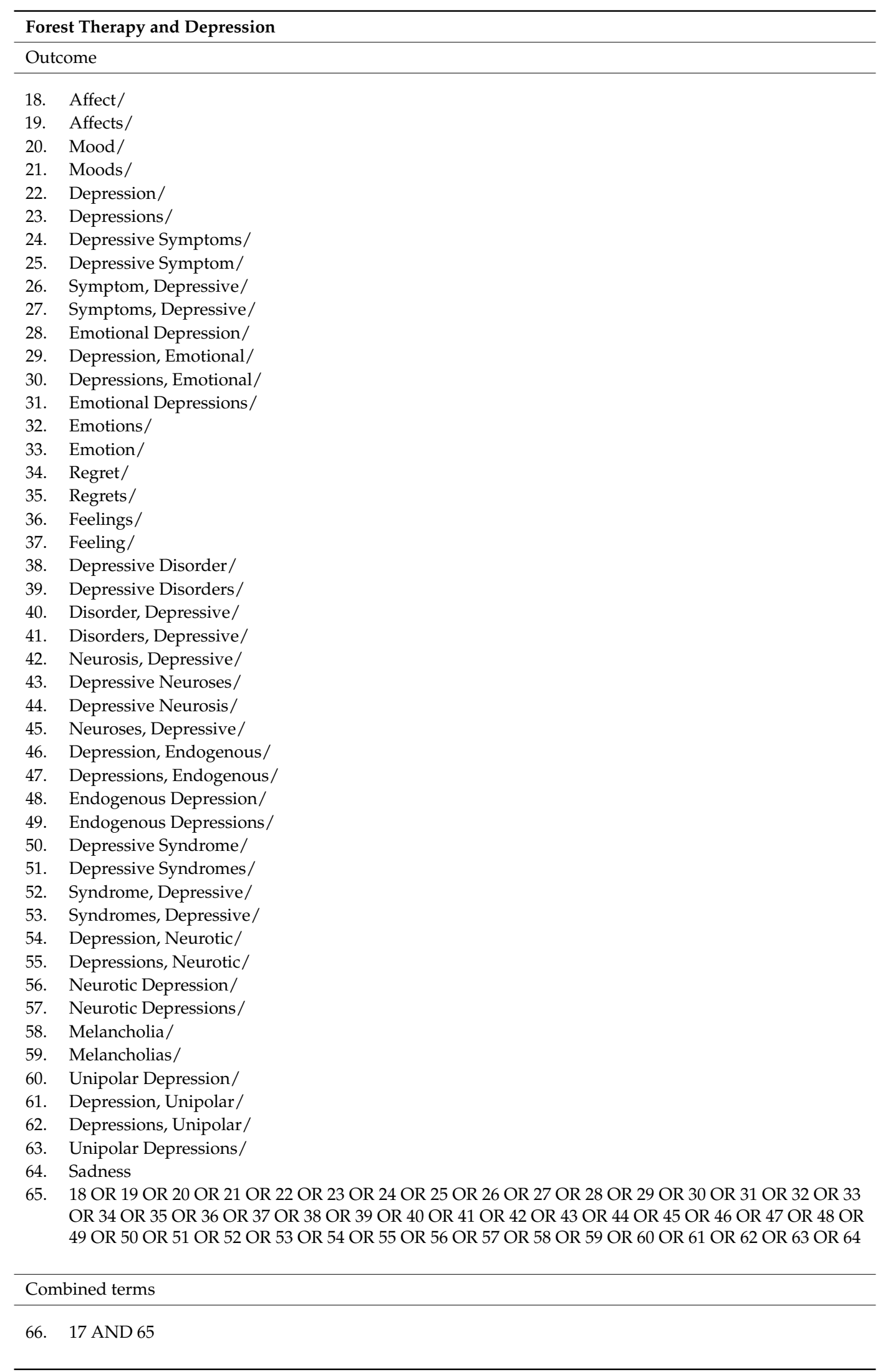




\section{References}

1. Li, Q. What Is Forest Medicine? In Forest Medicine; Nova Science Publisher: New York, NY, USA, 2013; pp. 3-10.

2. Park, B.J.; Tsunetsugu, Y.; Kasetani, T.; Hirano, H.; Kagawa, T.; Sato, M.; Miyazaki, Y. Physiological effects of shinrin-yoku (taking in the atmosphere of the forest)-Using salivary cortisol and cerebral activity as indicators. J. Physiol. Anthropol. 2007, 26, 123-128. [CrossRef] [PubMed]

3. Nilsson, U.; Fahlvik, N.; Johansson, U.; Lundström, A.; Rosvall, O. Simulation of the effect of intensive forest management on forest production in sweden. Forests 2011, 2, 373-393. [CrossRef]

4. Berman, M.G.; Jonides, J.; Kaplan, S. The cognitive benefits of interacting with nature. Psychol. Sci. 2008, 19, 1207-1212. [CrossRef] [PubMed]

5. Peen, J.; Schoevers, R.; Beekman, A.; Dekker, J. The current status of urban-rural differences in psychiatric disorders. Acta Psychiatr. Scand. 2010, 121, 84-93. [CrossRef] [PubMed]

6. Van Den Berg, A.E.; Maas, J.; Verheij, R.A.; Groenewegen, P.P. Green space as a buffer between stressful life events and health. Soc. Sci. Med. 2010, 70, 1203-1210. [CrossRef] [PubMed]

7. Li, Q.; Kobayashi, M.; Wakayama, Y. Effect of Forest Environments on Psychological Response Evaluated by the Poms Test. In Forest Medicine; Li, Q., Ed.; Nova Science Publisher: New York, NY, USA, 2013; pp. 137-146.

8. Higgins, J.P.; Green, S. Cochrane Handbook for Systematic Reviews of Interventions the Cochrane Collaboration: 2011. Available online: www.handbook.cochrane.org (accessed on 1 December 2016).

9. Crombie, I.K.; Davies, H.T. What Is Meta-Analysis. Available online: http://epu.ucc.ie/greid/ PL1025-PL1011-EH1008/Lectures/Term_2/DH_small_group_sessions/Fluoride/Meta-An.pdf (accessed on 1 December 2016).

10. World Health Organization. Depression Fact Sheet n 369. Available online: http://www.who.int/ mediacentre/factsheets/fs369/en/\# (accessed on 1 December 2016).

11. Petrie, J.; Grimshaw, J.; Bryson, A. The Scottish intercollegiate guidelines network initiative: Getting validated guidelines into local practice. Health Bull. 1995, 53, 345-348.

12. Barton, J.; Griffin, M.; Pretty, J. Exercise-, nature- and socially interactive-based initiatives improve mood and self-esteem in the clinical population. Perspect. Public Health 2012, 132, 89-96. [CrossRef] [PubMed]

13. Hong, S.S.; Kim, H.C.; Cho, S.H. The effects of forests healing for cognitive function. J. Orient. Neuropsychiatry 2013, 24, 63-74. [CrossRef]

14. Hong, S.S.; Lee, J.E.; Kim, H.C.; Cho, S.H. The effects of forests healing for hwa-byung. J. Orient. Neuropsychiatry 2012, 23, 169-182. [CrossRef]

15. Kim, M.H.; Shim, B.S.; Wi, A.J.; Yoon, B.S.; Han, Y.H.; Oh, E.M.; An, K.W. The influence of forest experience program on physiological and psychological states in psychiatric inpatients. J. Korean For. Soc. 2015, 104, 133-139. [CrossRef]

16. Shin, W.S.; Shin, C.S.; Yeoun, P.S. The influence of forest therapy camp on depression in alcoholics. Environ. Health Prev. Med. 2012, 17, 73-76. [CrossRef]

17. Woo, J.M.; Park, S.M.; Lim, S.K.; Kim, W. Synergistic effect of forest environment and therapeutic program for the treatment of depression. J. Korean For. Soc. 2012, 101, 677-685.

18. Bang, K.S.; Lee, I.S.; Kim, S.J.; Song, M.K.; Park, S.E. The effects of urban forest-walking program on health promotion behavior, physical health, depression, and quality of life: A randomized controlled trial of office-workers. J. Korean Acad. Nurs. 2016, 46, 140-148. [CrossRef] [PubMed]

19. Mao, G.X.; Lan, X.G.; Cao, Y.B.; Chen, Z.M.; He, Z.H.; Lv, Y.D.; Wang, Y.Z.; Hu, X.L.; Wang, G.F.; Yan, J. Effects of short-term forest bathing on human health in a broad-leaved evergreen forest in Zhejiang province, China. Biomed. Environ. Sci. 2012, 25, 317-324. [PubMed]

20. Chun, M.H.; Chang, M.C.; Lee, S.J. The effects of forest therapy on depression and anxiety in patients with chronic stroke. Int. J. Neurosci. 2017, 127, 199-203. [CrossRef] [PubMed]

21. Jia, B.B.; Yang, Z.X.; Mao, G.X.; Lyu, Y.D.; Wen, X.L.; Xu, W.H.; Lyu, X.L.; Cao, Y.B.; Wang, G.F. Health effect of forest bathing trip on elderly patients with chronic obstructive pulmonary diseas. Biomed. Environ. Sci. 2016, 29, 212-218. [PubMed]

22. Mao, G.X.; Cao, Y.B.; Lan, X.G.; He, Z.H.; Chen, Z.M.; Wang, Y.Z.; Hu, X.L.; Lv, Y.D.; Wang, G.F.; Yan, J. Therapeutic effect of forest bathing on human hypertension in the elderly. J. Cardiol. 2012, 60, 495-502. [CrossRef] [PubMed] 
23. Song, C.; Lee, J.; Ikei, H.; Kagawa, T.; Miyazaki, Y.; Park, B.-J. Physiological and psychological effects of walking around and viewing a lake in a forest environment. J. Korean For. Soc. 2015, 104, 140-149. [CrossRef]

24. Han, J.W.; Choi, H.; Jeon, Y.H.; Yoon, C.H.; Woo, J.M.; Kim, W. The effects of forest therapy on coping with chronic widespread pain: Physiological and psychological differences between participants in a forest therapy program and a control group. Int. J. Environ. Res. Public Health 2016, 13, 255. [CrossRef] [PubMed]

25. Horiuchi, M.; Endo, J.; Takayama, N.; Murase, K.; Nishiyama, N.; Saito, H.; Fujiwara, A. Impact of viewing vs. Not viewing a real forest on physiological and psychological responses in the same setting. Int. J. Environ. Res. Public Health 2014, 11, 10883-10901. [CrossRef] [PubMed]

26. Ji, G.B.; Kim, K.N.; Han, G.S. Physiological and psychological effects of viewing and walking in forest and urban area. J. Environ. Sci. Int. 2012, 21, 605-611. [CrossRef]

27. Kim, K.M.; Shin, W.S.; Park, B.J.; Oh, D.K. The psychological relaxation effects on forest space type. J. KIFR 2012, 16, 87-92.

28. Lee, J.; Park, B.J.; Tsunetsugu, Y.; Ohira, T.; Kagawa, T.; Miyazaki, Y. Effect of forest bathing on physiological and psychological responses in young Japanese male subjects. Public Health 2011, 125, 93-100. [CrossRef] [PubMed]

29. Lee, J.; Tsunetsugu, Y.; Takayama, N.; Park, B.J.; Li, Q.; Song, C.; Komatsu, M.; Ikei, H.; Tyrväinen, L.; Kagawa, T.; et al. Influence of forest therapy on cardiovascular relaxation in young adults. Evid.-Based Complement. Altern. Med. 2014, 2014, 834360. [CrossRef] [PubMed]

30. Lim, Y.S.; Kim, D.J.; Yeoun, P.S. Changes in depression degree and self-esteem of senior citizens in a nursing home according to forest therapy program. J. KIFR 2014, 18, 1-11.

31. Shin, W.S.; Oh, H.K. The influence of the forest program on depression level. J. Korean For. Soc. 1996, 85, 586-594.

32. Song, C.R.; Lee, J.Y.; Park, B.J.; Lee, M.S.; Matsuba, N.; Miyazaki, Y. Psychological effects of walking in the urban forests: Results of field tests in Shinjuku-Gyoen, Japan. J. Korean For. Soc. 2011, 100, 344-351.

33. Song, J.; Cha, J.; Lee, C.; Choi, Y.; Yeoun, P. Effects of forest healing program on stress response and spirituality in female nursing college students and there experience. J. KIFR 2014, 18, 109-125.

34. Takayama, N.; Korpela, K.; Lee, J.; Morikawa, T.; Tsunetsugu, Y.; Park, B.J.; Li, Q.; Tyrväinen, L.; Miyazaki, Y.; Kagawa, T. Emotional, restorative and vitalizing effects of forest and urban environments at four sites in Japan. Int. J. Environ. Res. Public Health 2014, 11, 7207-7230. [CrossRef] [PubMed]

35. Yang, S.S.; Cha, J.G.; Kim, J.A.; Hong, S.J.; Choi, Y.S. The effects of forest therapy program on spiritual health, self esteem depression and forest effectiveness in alcoholics' families. J. Korean Alcohol. Sci. 2011, 12, 45-59.

36. You, Y.S.; Lee, C.J.; Jan, N.C.; Son, B.K. A study of effects of sallimyok (forest therapy)-based mental health program on the depression the psychological stability. J. Korean Soc. Sch. Comm. Health Educ. 2014, 15, 55-65.

37. Choi, Y.H.; Ha, Y.S. The effectiveness of a forest-experience-integration intervention for community dwelling cancer patients' depression and resilience. J. Korean Acad. Community Health Nurs. 2014, 25, 109-118. [CrossRef]

38. Kim, Y.G.; Lee, S.H.; Kim, Y.H.; Eum, J.O.; Yim, Y.R.; Ha, T.G.; Shin, C.S. The influence of forest activity intervention on anxiety, depression, profile of mood states (POMS) and hope of cancer patients. J. KIFR 2015, 19, 65-74.

39. Song, C.; Ikei, H.; Kobayashi, M.; Miura, T.; Taue, M.; Kagawa, T.; Li, Q.; Kumeda, S.; Imai, M.; Miyazaki, Y. Effect of forest walking on autonomic nervous system activity in middle-aged hypertensive individuals: A pilot study. Int. J. Environ. Res. Public Health 2015, 12, 2687-2699. [CrossRef] [PubMed]

40. Lee, I.; Kim, S.; Bang, K.-S.; Choi, H.; Ko, C.; Jieun, K.; Sunyoung, K. An analysis of health promotion programs utilizing forests based on Korea's regional healthcare program plans. Perspect. Nurs. Sci. 2014, 11, 10-17. [CrossRef]

41. Stigsdotter, U.K.; Palsdottir, A.M.; Burls, A.; Chermaz, A.; Ferrini, F.; Grahn, P. Nature-based therapeutic interventions. In Forests, Trees and Human Health; Nilsson, K., Sangster, M., Gallis, C., Hartig, T., Vries, S.D., Seeland, K., Schipperijn, J., Eds.; Springer: New York, NY, USA, 2011; pp. 309-342.

42. Brunoni, A.R. Ceiling effects in the "effectiveness of adjunctive antidepressant treatment for bipolar depression" study: Was the sky the limit? Rev. Bras. Psiquiatr. 2011, 33, 102-103. [CrossRef] [PubMed]

43. Velengtas, P.; Mohr, P.; Messner, D.A. Making Informed Decisions: Assessing the Strengths and Weaknesses of Study Designs and Analytic Methods for Comparative Effectiveness Research. National Pharmaceutical Council, 2012. Available online: http:/ / www.ebmnow.org (accessed on 25 January 2017). 
44. Charness, G.; Gneezy, U.; Kuhn, M.A. Experimental methods: Between-subject and within-subject design. J. Econ. Behav. Organ. 2012, 81, 1-8. [CrossRef]

45. Grove, S.K.; Burns, N.; Gray, J. The Practice of Nursing Research: Appraisal, Synthesis, and Generation of Evidence, 7th ed.; Elsevier Health Sciences: St. Louis, MO, USA, 2012.

46. Polit, D.F.; Beck, C.T. Nursing Research: Generating and Assessing Evidence for Nursing Practice, 9th ed.; Wolters Kluwer Health: Philadelphia, PA, USA, 2008.

47. Blackhart, G.C.; Minnix, J.A.; Kline, J.P. Can eeg asymmetry patterns predict future development of anxiety and depression? A preliminary study. Biol. Psychol. 2006, 72, 46-50. [CrossRef] [PubMed]

48. Thibodeau, R.; Jorgensen, R.S.; Kim, S. Depression, anxiety, and resting frontal eeg asymmetry: A meta-analytic review. J. Abnorm. Psychol. 2006, 115, 715-729. [CrossRef] [PubMed]

49. Friedman, B.H.; Thayer, J.F. Autonomic balance revisited: Panic anxiety and heart rate variability. J. Psychosom. Res. 1998, 44, 133-151. [CrossRef]

50. Kim, G.M.; Woo, J.M. Determinants for heart rate variability in a normal Korean population. J. Korean Med. Sci. 2011, 26, 1293-1298. [CrossRef] [PubMed]

51. Kemp, A.H.; Quintana, D.S.; Felmingham, K.L.; Matthews, S.; Jelinek, H.F. Depression, comorbid anxiety disorders, and heart rate variability in physically healthy, unmedicated patients: Implications for cardiovascular risk. PLoS ONE 2012, 7, e30777. [CrossRef] [PubMed]

52. Stein, P.K.; Carney, R.M.; Freedland, K.E.; Skala, J.A.; Jaffe, A.S.; Kleiger, R.E.; Rottman, J.N. Severe depression is associated with markedly reduced heart rate variability in patients with stable coronary heart disease. J. Psychosom. Res. 2000, 48, 493-500. [CrossRef]

53. Levy, S.; Herberman, R.; Lippman, M.; D’Angelo, T. Correlation of stress factors with sustained depression of natural killer cell activity and predicted prognosis in patients with breast cancer. J. Clin. Oncol. 1987, 5, 348-353. [CrossRef] [PubMed]

54. Li, T.; Gleeson, $\mathrm{M}$. The effect of single and repeated bouts of prolonged cycling and circadian variation on saliva flow rate, immunoglobulin a and-amylase responses. J. Sports Sci. 2004, 22, 1015-1024. [CrossRef] [PubMed]

55. Skosnik, P.D.; Chatterton, R.T.; Swisher, T.; Park, S. Modulation of attentional inhibition by norepinephrine and cortisol after psychological stress. Int. J. Psychophysiol. 2000, 36, 59-68. [CrossRef]

56. Park, B.J.; Tsunetsugu, Y.; Lee, J.; Ishii, H.; Kagawa, T.; Miyazaki, Y. Effect of the forest environment on physiological relaxation using the results of field tests at 35 throughout japan. In Forest Medicine; Li, Q., Ed.; Nova Science Publisher: New York, NY, USA, 2013; pp. 57-67.

57. Li, Q.; Kobayashi, M.; Kawada, T. Carbamate pesticide-induced apoptosis and necrosis in human natural killer cells. J. Biol. Regul. Homeost. Agents 2013, 28, 23-32.

58. Olbrich, S.; Arns, M. Eeg biomarkers in major depressive disorder: Discriminative power and prediction of treatment response. Int. Rev. Psychiatry 2013, 25, 604-6189. [CrossRef] [PubMed] 